\title{
The Habit Tic: Onychotillomania
}

\author{
Shaheen Najafi, MD, Paul Aronowitz, MD, and George R. Thompson III, MD
}

Department of Medicine, Sacramento, CA, USA.

J Gen Intern Med 30(2):264

DOI: $10.1007 /$ s11606-014-2991-x

(c) Society of General Internal Medicine 2014

\section{CASE}

A 67-year-old man presented to his primary care physician for routine care. The absence of cuticles was noted on his bilateral thumbnails, and a midline crease with horizontal ridges from the proximal nail fold to the distal nail (Fig. 1). The patient stated that these changes had been present for over a year. He

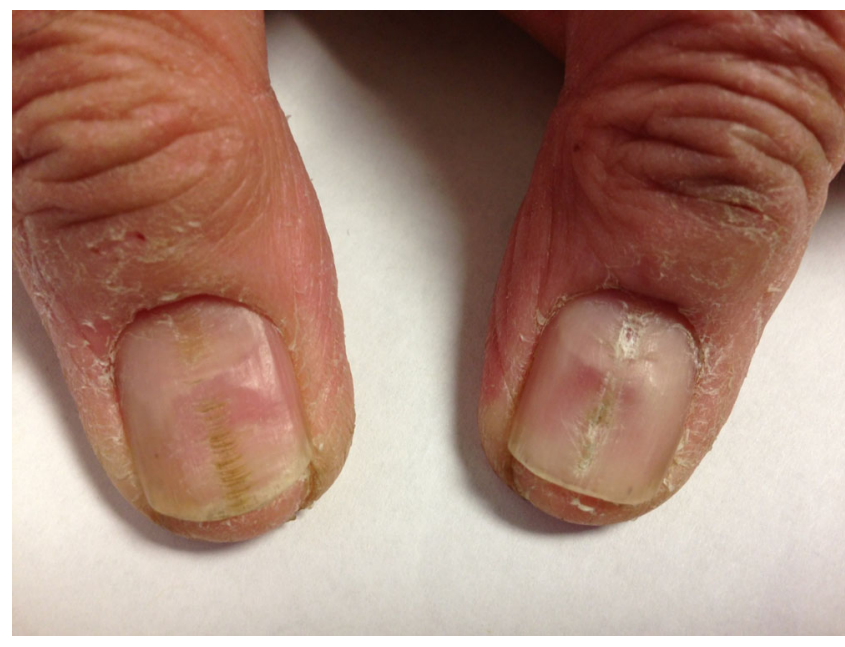

Figure 1. Nail changes of onychotillomania. Note the midline furrow on the nail plate, with a series of transverse ridges. admitted to frequent "picking" and biting of the cuticles and repeated hand washing, as evidenced by the xerosis of his fingers.

Habit-tic deformity is a type of onychodystrophy that is classified in a group of disorders known as onychotillomania, which includes entities such as nail biting (onychophagia), nail picking, and finger sucking. ${ }^{1}$ The deformity is the result of repetitive nail bed trauma from the conscious or unconscious manipulation of the cuticle. The characteristic findings include a midline furrow in the nail plate, often yellow in color, running the length of the nail, with a series of transverse ridges. ${ }^{2}$ Treatment includes physically covering the nails with bandages or glue to prevent further trauma. In addition, behavioral therapy and SSRIs have been tried, given the obsessive-compulsive nature of the habit. ${ }^{3,4}$ Cessation of the habit leads to complete resolution of the nail changes.

Corresponding Author: George R. Thompson, III, MD; Department of Medicine, 4150 V Street, Suite 2400, Sacramento, CA 95817 , USA (e-mail: grthompson@ucdavis.edu).

\section{REFERENCES}

1. Reese JM, Hudacek KD, Rubin AI. Onychotillomania: clinicopathologic correlations. J Cutan Pathol. 2013;40(4):419-23.

2. Ring DS. Inexpensive solution for habit-tic deformity. Arch Dermatol. 2010;146(11):1222-3.

3. Vittorio CC, Phillips KA. Treatment of habit-tic deformity with fluoxetine. Arch Dermatol. 1997;133(10):1203-4.

4. Gloster $\mathbf{H}$ Jr, Kindred C. Habit-tic-like and median nail-like dystrophies treated with multivitamins. J Am Acad Dermatol. 2005;53(3):543-4. 